\title{
Time dependent quantum graph with loop
}

\author{
D. A. Eremin ${ }^{1}$, E. N. Grishanov ${ }^{1}$, O. G. Kostrov ${ }^{1}$, D. S. Nikiforov ${ }^{2}$, I. Y. Popov ${ }^{2}$ \\ ${ }^{1}$ Department of Mathematics and IT, Ogarev Mordovia State University \\ Bolshevistskaya Str. 68, Saransk, Russia \\ ${ }^{2}$ Department of Higher Mathematics, ITMO University, Kroverkskiy pr. 49, St. Petersburg, 197101, Russia \\ popov1955@gmail.com
}

\begin{abstract}
A quantum graph, consisting of a ring and segment is considered. We deal with the free Schrödinger ooperator at the edges and Kirchhoff conditions at the internal vertex. The lengths of the graph edges varies in time. Time evolution of wave packet is studied for different
\end{abstract} parameters of length varying law.

Keywords: quantum graph, spectrum, time evolution.

Received: 26 May 2017

Revised: 20 June 2017

\section{Introduction}

A quantum graph is a rather old mathematical model introduced initially to aid in the description of macromolecules [1]. Later, it was used in many problems of quantum theory. In spite of its simplicity, the model gives one a powerful instrument for investigating quantum systems. As for rigorous mathematical models of such type, they appeared in the 80's [2]. The mathematical background of the approach is the theory of self-adjoint operator extensions (see, e.g., [3,4] and references in [5]) related to model of point-like potentials in quantum physics. There are a large number of works in the field (see, e.g., [6-9] and references therein).

The problem of time-dependent boundary conditions in the Schrödinger equation has attracted much attention in the context of quantum Fermi acceleration [10]. A detailed study of the problem can be found in [11]. In particular, it was pointed out that the problem of $1 \mathrm{D}$ box with a moving wall can be mapped onto that of an harmonic oscillator with time-dependent frequency confined inside the static box. Time-dependent point-like potentials and related topics of the operator extensions theory were studied in several works (see, e.g., [12] and references therein). Star-like quantum graphs having edges with time varying lengths have been discussed [13]. Problems concerning the boundary conditions for time-depended interval are discussed in [14].

In the present paper, we consider simple quantum graph with a loop (see Fig. 1). The lengths of edges vary in time. We construct a mathematical model and investigate the time evolution of initial wave packet.

\section{The description of the model}

\subsection{Stationary problem}

To describe the system evolution, we start from the stationary problem. The quantum graph is determined in a conventional way. The Hamiltonian is the free Schrödinger operator, i.e. $-\frac{d^{2}}{d y^{2}}$, at each edge and the Kircchoff conditions at the internal vertex of the graph. The length of the segment is assumed to be 1 , the circle radius is $r$. Let us mark the electron wave function as $\phi_{\ell}$ at the segment and as $\phi_{r}$ at the ring. Then, one has the following equation at each edge:

$$
\begin{gathered}
-\frac{d^{2}}{d y^{2}} \phi_{\ell}(y)=k^{2} \phi_{\ell}(y), 0 \leq y \leq 1, \\
-\frac{d^{2}}{d y^{2}} \phi_{r}(y)=k^{2} \phi_{r}(y), 0 \leq y \leq 2 \pi r, \\
\left\{\begin{array}{l}
\phi_{\ell}(0)=\phi_{r}(0)=\phi_{r}(2 \pi r), \\
\phi_{\ell}(\ell)=0, \\
\left.\frac{d}{d y} \phi_{l}\right|_{y=0}+\left.\frac{d}{d y} \phi_{r}\right|_{y=0}-\left.\frac{d}{d y} \phi_{r}\right|_{y=2 \pi r}=0 .
\end{array}\right.
\end{gathered}
$$




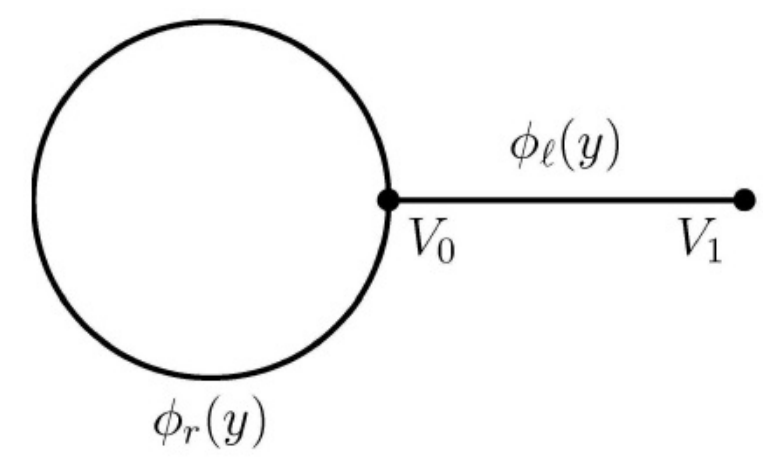

FIG. 1. Graph geometry

As for the boundary vertex of the graph, we assume here Dirichlet conditions. Eigenfunctions take the form:

$$
\begin{aligned}
\phi_{\ell}^{(n)}(y) & =\frac{1}{B_{n}} \frac{\sin \left(k_{n}(1-y)\right)}{\sin \left(k_{n}\right)}, \\
\phi_{r}^{(n)}(y) & =\frac{1}{B_{n}} \frac{\cos \left(k_{n}(y-\pi r)\right)}{\cos \left(k_{n} \pi r\right)},
\end{aligned}
$$

where $k_{n}$ is the $n$-th positive root of the spectral equation having the form:

$$
2 \tan (\pi k r)=\cot k \text {. }
$$

$B_{n}$ is the normalizing constant:

$$
B_{n}^{2}=\frac{1}{2 \sin ^{2}\left(k_{n}\right)}+\frac{\pi r}{\cos ^{2}\left(\pi k_{n} r\right)}
$$

\subsection{Non-stationary problem}

Let us consider a non-stationary graph. We assume that the edge lengths vary with time, $L_{\ell}=L(t)$, $L_{r}=2 \pi r L(t)$. Here, $L(t)$ is a smooth positive function (we will choose it later). In this case, the particle dynamics in graph are described by the following time-dependent Schrödinger equation:

$$
\begin{aligned}
\imath \frac{\partial}{\partial t} \Psi_{\ell}(x, t) & =-\frac{\partial^{2}}{\partial x^{2}} \Psi_{\ell}(x, t), 0 \leq x \leq L(t), \\
\imath \frac{\partial}{\partial t} \Psi_{r}(x, t) & =-\frac{\partial^{2}}{\partial x^{2}} \Psi_{r}(x, t), 0 \leq x \leq 2 \pi r L(t),
\end{aligned}
$$

for the time-dependent wave function:

$$
\Psi(t)=\left(\begin{array}{c}
\Psi_{\ell}(x, t) \\
\Psi_{r}(x, t)
\end{array}\right) .
$$

Here, the first argument of $\Psi_{\ell}(x, t)$ varies as follows $0 \leq x \leq L(t)$ and the first argument of $\Psi_{r}(x, t)-$ in $0 \leq x \leq 2 \pi r L(t)$. We choose the system of units in which the Planck's constant, speed of light and the electron mass are as follows: $\hbar=c=1, m=1 / 2$. The following coupling conditions (Kircchoff conditions) take place at the internal vertex $V_{0}$ of the graph and the Dirichlet condition at vertex $V_{1}$ :

$$
\left\{\begin{array}{l}
\left.\Psi_{\ell}\right|_{x=0}=\left.\Psi_{r}\right|_{\varphi=0}=\left.\Psi_{r}\right|_{\varphi=2 \pi r}, \\
\Psi_{\ell}(L(t))=0, \\
\left.\frac{\partial}{\partial x} \Psi_{\ell}\right|_{x=0}+\left.\frac{\partial}{\partial x} \Psi_{r}\right|_{x=0}-\left.\frac{\partial}{\partial x} \Psi_{r}\right|_{x=2 \pi r}=0 .
\end{array}\right.
$$

Let us replace the variables at the edges: $y=\frac{x}{L(t)}$. Then, the equations change (we have the same equation both at the segment and at the ring, they differ only in variables range):

$$
\begin{array}{ll}
\imath \frac{\partial}{\partial t} \Psi_{\ell}(y, t)=-\frac{1}{L^{2}(t)} \frac{\partial^{2}}{\partial y^{2}} \Psi_{\ell}(y, t)+\imath \frac{\dot{L}(t)}{L(t)} y \frac{\partial}{\partial y} \Psi_{\ell}(y, t), & 0 \leq y \leq 1, \\
\imath \frac{\partial}{\partial t} \Psi_{r}(y, t)=-\frac{1}{L^{2}(t)} \frac{\partial^{2}}{\partial y^{2}} \Psi_{r}(y, t)+\imath \frac{\dot{L}(t)}{L(t)} y \frac{\partial}{\partial y} \Psi_{r}(y, t), & 0 \leq y \leq 2 \pi r .
\end{array}
$$


To obtain a self-adjoint problem, we make the following replacement:

$$
\Psi(t)=\left(\begin{array}{c}
\Psi_{\ell}(y, t) \\
\Psi_{r}(y, t)
\end{array}\right)=\frac{1}{\sqrt{L(t)}} e^{\imath \frac{L \dot{L}}{4} y^{2}}\left(\begin{array}{c}
\psi_{\ell}(y, t) \\
\psi_{r}(y, t)
\end{array}\right)
$$

Correspondingly, one has the following equations for functions $\psi_{\ell}(y, t), \psi_{r}(y, t)$ :

$$
\begin{array}{ll}
\imath \frac{\partial}{\partial t} \psi_{\ell}(y, t)=-\frac{1}{L^{2}(t)} \frac{\partial^{2}}{\partial y^{2}} \psi_{\ell}(y, t)+\frac{L \ddot{L}}{4} y^{2} \psi_{\ell}(y, t), \quad 0 \leq y \leq 1, \\
\imath \frac{\partial}{\partial t} \psi_{r}(y, t)=-\frac{1}{L^{2}(t)} \frac{\partial^{2}}{\partial y^{2}} \psi_{r}(y, t)+\frac{L \ddot{L}}{4} y^{2} \psi_{\ell}(y, t), \quad 0 \leq y \leq 2 \pi r .
\end{array}
$$

One can see that the Kirchoff conditions (1) remain for functions $\psi_{\ell}(y, t), \psi_{r}(y, t)$ at vertex $V_{0}$ and the Dirichlet conditions at $V_{1}$.

Taking into account that now the geometric graph is stationary after the chang of variables, we use the expansions with respect to complete system of orthogonal and normalized eigenfunctions of the self-adjoint operator (see Section 2):

$$
\left(\begin{array}{c}
\psi_{\ell}(y, t) \\
\psi_{r}(y, t)
\end{array}\right)=\sum_{n} C_{n}(t)\left(\begin{array}{c}
\phi_{\ell}^{(n)}(y) \\
\phi_{r}^{(n)}(y)
\end{array}\right)
$$

Let us insert (3) into (2) and (3). We obtain the system for coefficients $C_{n}$ :

$$
\imath_{i}(t)=-\frac{k_{m}^{2}}{L^{2}} C_{m}(t)+\sum_{n} M_{m n} C_{n}(t),
$$

where:

$$
M_{m n}=\frac{L \ddot{L}}{4}\left(\int_{0}^{1} y^{2} \phi_{\ell}^{(n)}(y) \overline{\phi_{\ell}^{(m)}(y)} d y+\int_{0}^{2 \pi r} y^{2} \phi_{r}^{(n)}(y) \overline{\phi_{r}^{(m)}(y)} d y\right) .
$$

This is an infinite system. We truncate it and numerically solve the obtained finite-size system. To consider the evolution of wave packet, we realize the following procedure: we take some initial value for the wave function, expand it in a series, truncate the series, solve the system for coefficients and summarize the series with coefficients corresponding to chosen time value. Correspondingly, we choose the initial condition:

$$
\Psi(0)=\left(\begin{array}{c}
\Psi_{l}(x, 0) \\
\Psi_{r}(x, 0)
\end{array}\right) .
$$

The initial values for $\psi_{\ell}(y, t), \psi_{r}(y, t)$ are obtained in the following way:

$$
\left(\begin{array}{c}
\psi_{\ell}(y, 0) \\
\psi_{r}(y, 0)
\end{array}\right)=\left(\begin{array}{c}
L(0) e^{-\imath \frac{L(0) \dot{L}(0)}{4} y^{2}} \Psi_{\ell}(y, 0) \\
0
\end{array}\right) .
$$

Correspondingly, the initial values for our system of ordinary differential equation are as follows:

$$
C_{n}(0)=\int_{0}^{1} \psi_{\ell}(y, 0) \overline{\phi_{\ell}^{(n)}} d y
$$

The solution of the system (4) gives us values for $C_{n}(t)$. By inserting it into (3), we obtain the wave function for the moment $t$.

\section{Results and discussion}

We will choose the particular type of length variation, namely, the harmonic dependence:

$$
L(t)=a+b \cos (\omega t) .
$$

Here, $\omega$ is the frequency of the length vibration. We take the following initial wave function (concentrated at the segment):

$$
\Psi(0)=\left(\begin{array}{c}
\Psi_{l}(x, 0) \\
\Psi_{r}(x, 0)
\end{array}\right)=\left(\begin{array}{c}
(1-\cos (2 \pi x)) \sqrt{2 / 3} \\
0
\end{array}\right)
$$



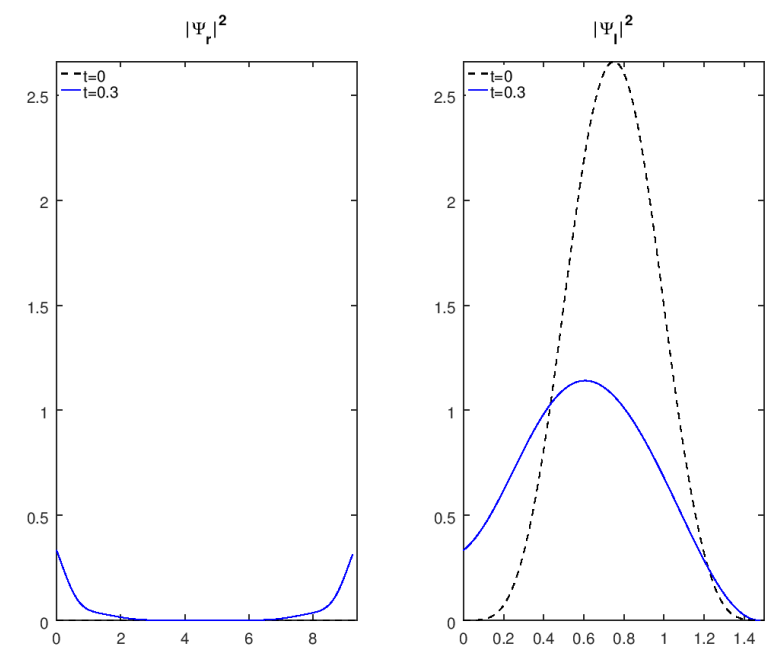

FIG. 2. The wave function at the ring (left) and segment (right) $t=0, t=0.3$ (arbitrary units)
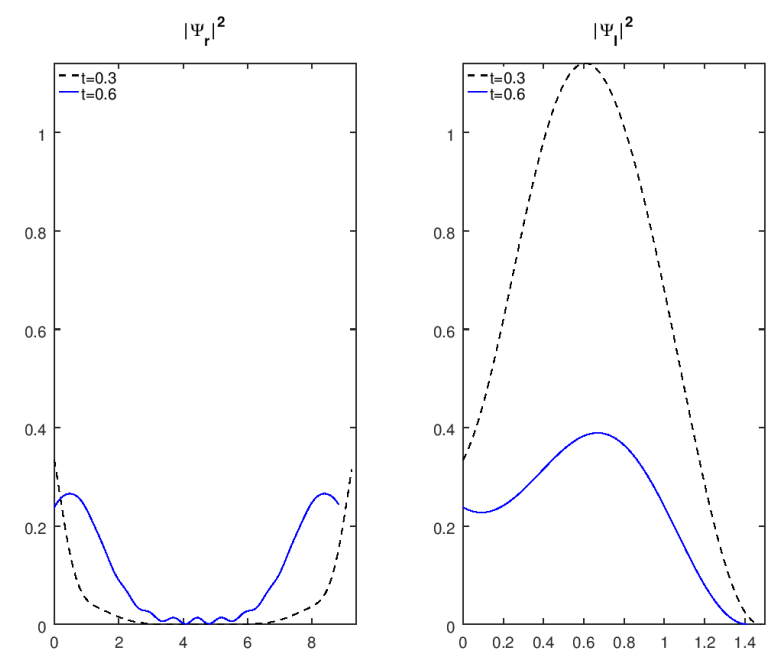

FIG. 3. The wave function at the ring (left) and segment (right) $t=0.3, t=0.6$ (arbitrary units)
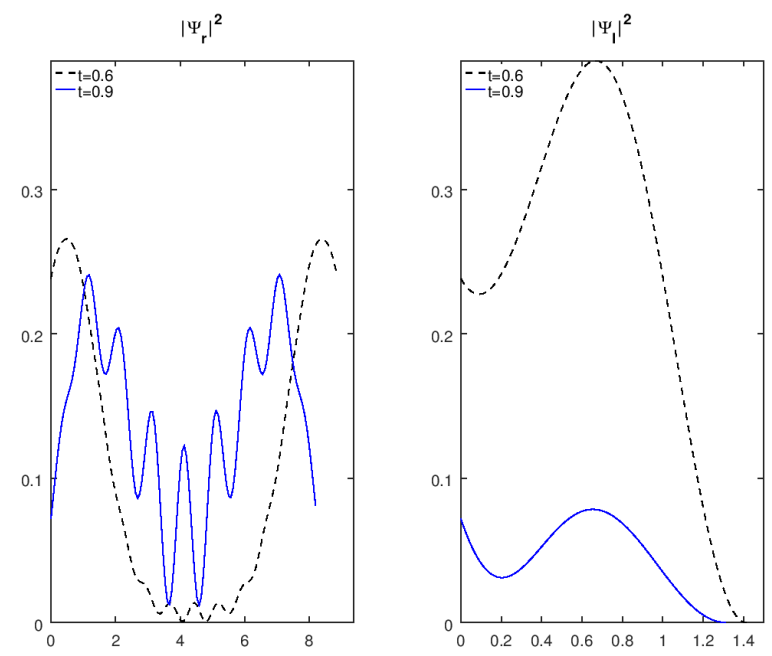

FIG. 4. The wave function at the ring (left) and segment (right) $t=0.6, t=0.9$ (arbitrary units) 

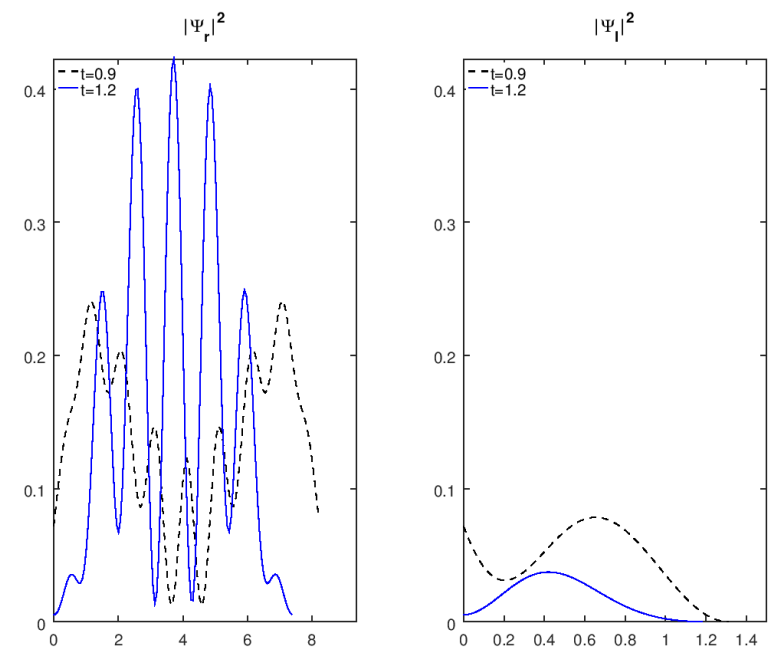

FIG. 5. The wave function at the ring (left) and segment (right) $t=0.9, t=1.2$ (arbitrary units)
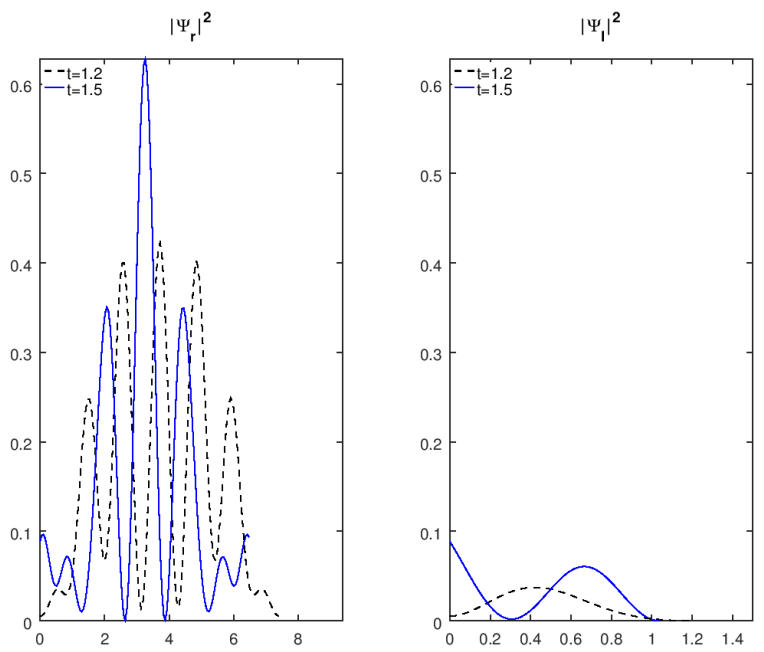

FIG. 6. The wave function at the ring (left) and segment (right) $t=1.2, t=1.5$ (arbitrary units)
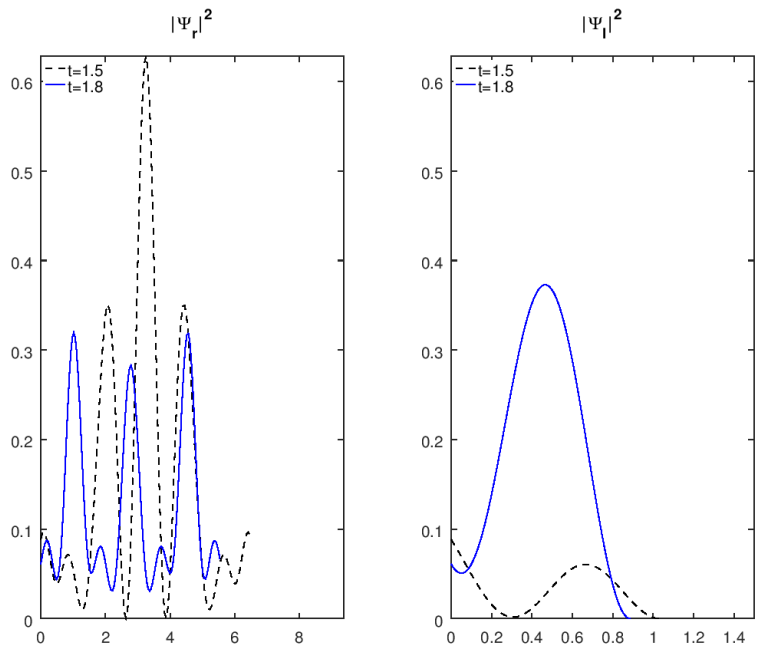

FIG. 7. The wave function at the ring (left) and segment (right) $t=1.5, t=1.8$ (arbitrary units) 
The wave function for the time moment $t$ is obtained by the procedure described in the previous section. The following parameters are chosen: $a=1, b=1 / 2, \omega=1, r=1$. Time evolution of the wave packet is shown in Figs. 2-7, corresponding to time values $t=0, t=1.1, t=1.3, t=1.4$ in initial coordinate system.

\section{Acknowledgements}

This work was partially financially supported by the Government of the Russian Federation (grant 074-U01), by grant MK-5161.2016.1 of the President of the Russian Federation, DFG Grant NE 1439/3-1, by grant 16-11-10330 of Russian Science Foundation.

\section{References}

[1] Pauling L. The Diamagnetic Anisotropy of Aromatic Molecules. J. Chem. Phys., 1936, 4, P. 673.

[2] Gerasimenko N.I., Pavlov B.S. Scattering problems on noncompact graphs. Theoret. Math. Phys., 1988, 74, P. $230-240$.

[3] Albeverio S., Gesztesy F., Hoegh-Krohn R., Holden H. with an appendix by P. Exner. Solvable Models in Quantum Mechanics: Second Edition, 2005. (Providence, R.I.: AMS Chelsea Publishing).

[4] Pavlov B.S. The theory of extensions and explicity-solvable models. Russ. Math. Surv., 1987, 42(6), P. 127-168.

[5] Popov I.Y., Kurasov P.A., Naboko S.N., Kiselev A.A., Ryzhkov A.E., Yafyasov A.M., Miroshnichenko G.P., Karpeshina Yu.E., Kruglov V.I., Pankratova T.F., Popov A.I. A distinguished mathematical physicist Boris S. Pavlov. Nanosystems: Physics, Chemistry, Mathematics, 2016, 7, P. 782-788.

[6] Exner P., Keating P., Kuchment P., Sumada T., Teplyaev A. Analysis on graph and its applications. Proc. Symp. Pure Math. Providence, RI, 2008, 77.

[7] Duclos P., Exner P., Turek O. On the spectrum of a bent chain graph. J. Phys. A: Math. Theor., 2008, 41, P. 415206/1-18.

[8] Popov I.Y., Skorynina A.N., Blinova I.V. On the existence of point spectrum for branching strips quantum graph. J. Math. Phys., 2014, 55, P. 033504/1-20.

[9] Popov I.Y., Smirnov P.I. Spectral problem for branching hain quantum graph. Phys. Lett., 2013, A 377, P. 439-442.

[10] Jose J.V., Gordery R. Study of a quantum Fermi-acceleration model. Phys. Rev. Lett., 1986, 56, P. 290.

[11] Makowski A.J., Dembinski S.T. Exactly solvable models with ime-dependent boundary conditions. Phys. Lett., 1991, A, 154(5-6), P. 217220.

[12] Cacciapuoti C., Mantile A., Posilicano A. Time dependent delta-prime interactions in dimension one. Nanosystems: Phys. Chem. Math., 2016, 7(2), P. 303-314.

[13] Matrasulov D.U., Yusupov J.R., Sabirov K.K., Sobirov Z.A. Time-dependent quantum graph. Nanosystems: Phys. Chem. Math., 2015, 6(2), P. 173-181.

[14] Karpova O., Sabirov K., Otajanov D., Ruzmetov A., Saidov A.A. Absorbing boundary conditions for Schrödinger equation in a timedependent interval. Nanosystems: Phys. Chem. Math., 2017, 8(1), P. 13-19. 